\title{
Measuring intraspecific variation in the response of clinical isolates of Aspergillus fumigatus to oxidative stress.
}

\section{Sam El-Kamand}

Western Sydney University - Campbelltown Campus

\section{Carl Ramirez}

Western Sydney University - Campbelltown Campus

Catriona Halliday

Westmead Hospital

Sharon C-A. Chen

Westmead Hospital

Charles Oliver Morton ( $\nabla$ o.morton@westernsydney.edu.au )

Western Sydney University https://orcid.org/0000-0003-1702-0545

\section{Research note}

Keywords: Aspergillus fumigatus, oxidative stress, intraspecific variation, pathogenicity

Posted Date: July 2nd, 2019

DOI: https://doi.org/10.21203/rs.2.10806/v1

License: (c) (i) This work is licensed under a Creative Commons Attribution 4.0 International License. Read Full License 


\section{Abstract}

Objective In this study, the survival of clinical isolates of the pathogenic fungus Aspergillus fumigatus against the oxidative stressors, hydrogen peroxide and menadione, and UV light, was examined to see if there was variation between isolates and if the variation was linked to virulence. Results Fifteen isolates were tested, five from cases of invasive aspergillosis (IA isolates) and ten from cases where the fungus had colonised a patient (colonising isolates). Exposure to UV light and hydrogen peroxide did not show significant differences between the groups of isolates. Colonising isolates showed a trend for greater survival when treated with hydrogen peroxide, mean survival $18.9 \%$, compared to IA isolates, mean survival $8 \%$. Treatment with $50 \mathrm{mM}$ menadione confirmed this trend in colonising isolates with an average conidial survival of $72 \%$ compared to $50 \%$ in IA isolates. Overall significant sensitivity to $50 \mathrm{mM}$ menadione was observed in 1/10 colonising isolates compared to 4/5 IA isolates. Increased sensitivity to oxidative stress in IA isolates may seem counterintuitive but could be utilised as an indicator of pathogenic potential in isolates of the fungus and be used in further studies to unravel the complex interplay between host and pathogen.

\section{Introduction}

The ascomycete fungus Aspergillus fumigatus is a globally distributed decomposer of organic matter in the environment. Its broad distribution is due to its production of vast numbers of conidia which are easily distributed by wind currents. These conidia are inhaled by birds and mammals in which it can cause several disease states (1). In humans the most severe disease state is invasive aspergillosis, which typically affects immunocompromised individuals particularly those that are neutropenic (2). For conidia to enter the host and lead to successful infection of a human they must overcome a number of challenges including; UV radiation during dispersal (3), innate immune effectors such as antimicrobial peptides and phagocytosis by immune cells including alveolar macrophages (4), localised hypoxic conditions (5), and essential nutrient acquisition (6).

Conidia and early developmental stages of the fungus also face a particular challenge with regard to oxidative stress. Exposure to UV light can induce the production of reactive oxygen species within cells (7), countered in conidia by their high melanin content where melanin also plays a role in overcoming host defences, by resisting the oxidative burst produced by immune cells and in modulating the internal environment within the phagolysosomes of alveolar macrophages (8).

Resistance to innate host defences has been proposed to be a significant factor in the establishment of IA. Like bacterial pathogens it is increasingly clear that not all isolates of a pathogenic fungi are equally virulent $(9,10)$. Studies have described differences between environmental and clinical isolates of $A$. fumigatus with respect to virulence in murine models through quantification of pulmonary fungal burden and measurement of mortality $(11,12)$. In this study we compared the response of clinical isolates of $A$. fumigatus from patients that had IA (termed "IA isolates"), and those that did not develop IA but were colonised by the fungus, (termed "colonising isolates"), to oxidative stress by measuring conidial survival 
in response to $\mathrm{H}_{2} \mathrm{O}_{2}$, menadione, and UV light. This may help to elucidate factors that may be relevant to the outcome of the host fungus interaction.

\section{Methods And Materials}

Organisms and strains: Fifteen strains of $A$. fumigatus were studied, and were obtained from the Centre of Infectious Diseases and Microbiology Laboratory Services, Westmead hospital. Ten (Af1 - Af10) were from patients colonised with A. fumigatus (colonising isolates) and five (Af11 -Af15), were from cases of proven IA (IA isolates). Cultures were grown on potato dextrose agar (PDA) for three days at 37C and conidia were isolated from each strain as previously described (13).

Measurement of response to hydrogen peroxide and menadione: To measure the effects of acute exposure to menadione ( $10 \mathrm{mM}$ stock in ethanol) and $\mathrm{H}_{2} \mathrm{O}_{2}$ (30\% solution) $A$. fumigatus spore suspensions from each isolate, $1 \times 10^{6}$ conidia/ml, were incubated for 3 hours at $37^{\circ} \mathrm{C}$ with $0 \mathrm{mM}, 30 \mathrm{mM}$ or $50 \mathrm{mM}$ of menadione or $\mathrm{H}_{2} \mathrm{O}_{2}$. After incubation conidia were dilution plated onto PDA and incubated at $37^{\circ} \mathrm{C}$ for 24 hours and counted to determine $\mathrm{CFU} / \mathrm{mL}$. The percent inhibition was calculated relative to the $0 \mathrm{mM}$ control.

Measurement of response to UV light: For each isolate, approximately 200 conidia were spread plated onto malt extract agar (MEA). Five plates inoculated with the same isolate were placed at different positions within a TopSafe PC2 Biosafety cabinet and UV irradiated $(1.6 \mathrm{~W} / \mathrm{m} 2)$ for 1 minute. This was repeated for all 15 isolates. Following irradiation of each isolate, the biosafety cabinet was vented for 5 minutes to prevent ROS accumulation. Colony forming units (CFU) on control plates were counted following incubation at $37^{\circ} \mathrm{C}$ for $24 \mathrm{~h}$. An additional incubation for $24 \mathrm{hrs}$ at $25^{\circ} \mathrm{C}$ preceded $\mathrm{CFU}$ counting of UV-irradiated plates. Percent survival for each isolate was calculated relative to a non-irradiated control and based on the average CFU counts across the five irradiated plates. The experiment was repeated four times, with the order in which isolates were irradiated changed to achieve uniform average UV-order position amongst all isolates.

Statistical Analysis: All data were analysed using Graphpad version 7, UV data was analysed using ANOVA and post-test (Dunn's) and menadione $/ \mathrm{H}_{2} \mathrm{O}_{2}$ data were analysed by t-test. There were five replicates of each experiment.

\section{Results And Discussion}

The effect of hydrogen peroxide and menadione on clinical isolates of A. fumigatus. The addition of 30 $\mathrm{mM} \mathrm{H}_{2} \mathrm{O}_{2}$ had a similar effect on all isolates tested, the average survival for colonising isolates was $58 \%$ and survival for $\mathrm{IA}$ isolates was $59 \%$. Increasing $\mathrm{H}_{2} \mathrm{O}_{2}$ to $50 \mathrm{mM}$ had a greater effect on survival and revealed greater differences between isolates, the average survival for colonising isolates was $18.9 \%$ and survival for IA isolates $8 \%$. These broad average values suggest a difference between the two groups of isolates but the greater value for coloniser was caused by higher survival rates in just four isolates Af01 - 
Af04. By comparing the results at $30 \mathrm{mM}$ and $50 \mathrm{mM}$ only one isolate, showed statistically significant resistance to $\mathrm{H}_{2} \mathrm{O}_{2}$ (Table 1).

The data for menadione indicate that it has a smaller effect on survival with increasing doses, this created the subtlety necessary to discriminate between isolates in terms of sensitivity or resistance to oxidative stress (Table 1). Addition of $30 \mathrm{mM}$ menadione led to an average survival of $80 \%$ for colonising isolates and $76 \%$ for IA isolates. Addition of $50 \mathrm{mM}$ led to an average survival of $72 \%$ in colonising isolates and $50 \%$ in IA isolates. This is a more pronounced difference than for $\mathrm{H}_{2} \mathrm{O}_{2}$, the effect was not confined to a small number of isolates skewing the data, comparison of survival for each isolate at 30 $\mathrm{mM}$ and $50 \mathrm{mM}$ revealed that $90 \%$ of colonising isolates were relatively resistant to menadione whereas this was just $20 \%$ for IA isolates (Table 1). These data suggest that there is a difference in the ability of $A$. fumigatus isolated from patients where the fungus was just a coloniser compared to isolates that caused invasive disease with respect to tolerance of oxidative stress. However, this would require confirmation in larger scale studies.

The effect of acute exposure to UV on clinical isolates of A. fumigatus. There were only four isolates that were relatively resistant to UV light exposure and these showed $>45 \%$ survival, the most sensitive isolates showed $15 \%$ survival (Figure 1). Resistance was not associated with whether colonising (three isolates) or IA isolates (one isolate) were tested. These data do not suggest that there are any important differences between isolates with regard to UV sensitivity, there was no clustering of sensitive or resistant isolates based on origin of the isolate. UV resistance in conidia is related to the melanin in the conidial cell wall which mitigate UV damage to ensure spore survival (8). However, modulating UV intensity was not possible in our experimental setting and therefore we could not modify the experiment to detect suitably subtle variations in response of isolates to UV. An element of UV toxicity is related to the generation of oxidative stress in host cells; isolates Af01, Af04, Af09, and Af14 that showed relative resistance to UV were also resistant to treatment with $50 \mathrm{mM}$ menadione. However, only Af01 and Af04 showed resistance to $\mathrm{H}_{2} \mathrm{O}_{2}$ exposure. These suggest an overlap in resistance to UV damage and menadione. Studies in Saccharomyces cerevisiae indicated that different genes were induced during exposure to $\mathrm{H}_{2} \mathrm{O}_{2}$ compared to menadione (14) so it should be expected that isolates might display different survival characteristic when comparing both stressors.

Phenotypic variation and virulence. There have been studies that have compared the phenotypic characteristics of clinical and environmental isolates of $A$. fumigatus. It has been observed that the rate of radial growth on agar plates is correlated with virulence in $A$. fumigatus, with faster growing isolates being more virulent (15). Further, it has been found that cell wall chitin content and hyphal diameter in an environmental isolate led to decreased virulence in this isolate showing further characteristics that could be considered important in characterising virulent isolates (16). The key characteristic that has been used to compare isolates has been virulence in murine models of IA. In a study using mixed isolate infections it was found that the more virulent isolates had a clinical origin but the degree of virulence in the environmental isolates suggested that they would still have the capacity to cause infection (11). Another study tested the hypothesis that certain isolates of $A$. fumigatus are more virulent than others and was 
able to demonstrate this in a murine model, finding that environmental isolates were less virulent than clinical isolates (12). These studies support the findings that intraspecific variation in virulence exists in A. fumigatus but the mechanisms have been difficult to identify. This is a particular problem with an opportunistic pathogen, which does not possess a specific virulence mechanism.

The host response to $A$. fumigatus has been studied as a means to define pathogenicity by interacting the isolates of the fungus with monocyte-derived dendritic cells and mice (17). The study found that moDCs produced strain specific cytokine responses; it was further observed that different wild-type isolates of the fungus induced inflammatory or hyperinflammatory responses in immunocompetent mice and that these differences in inflammatory response had a strong effect on the outcome of infection (17). It may seem counterintuitive that the IA isolates showed reduced resistance to oxidative stress induced by menadione compared to colonising isolates but if host immune interactions determine the outcome of IA then factors linked to this reduced oxidative resistance may be crucial in the development of IA making these isolates of value in future host-interaction studies.

\section{Limitations}

Limitations of this research focus on the need to test a greater number of isolates from more clinical centres to have a true representation of the oxidative stress sensitivity in the species. Ideally, we could have used a greater variety of doses of $\mathrm{H}_{2} \mathrm{O}_{2}$ to find a concentration that could show variation between isolates. A further limitation is that it would have been ideal to have characterised the virulence of each strain of the fungus.

\section{List Of Abbreviations}

\section{$\mathrm{H}_{2} \mathrm{O}_{2}$, hydrogen peroxide. IA, invasive aspergillosis. UV, ultraviolet. PDA, potato dextrose agar. CFU, colony forming unit.}

\section{Declarations}

\section{Author's Contributions:}

SEM and CR performed the experimental work for the study. AP assisted in experimental design and preparation of the manuscript. $\mathrm{CH}$ and $\mathrm{SC}$ assisted in experimental design and preparation of the manuscript and provided the clinical isolates. COM designed the study, assisted in data analysis and wrote the manuscript. 


\section{Acknowledgements:}

The authors wish to acknowledge funding from Western Sydney University that enabled Sam El-Kamand and Carl Ramirez to undertake this research. The authors would also like to thank Karena Gilroy, Chun Ho, and Smitha Parameswaran for technical support.

\section{Author Information:}

SEM, CR, COM at Western Sydney University, School of Science and Health, Building 21 Campbelltown Campus, Narellan Road, NSW 2560, Australia. CH and SC at Westmead Hospital, ICPMR, Westmead, NSW Australia

\section{Competing interests:}

The authors declare that they have no competing interests that could affect the integrity of this study.

\section{Availability of Data and Material:}

The data supporting the results of this study are included within this article; raw data can be requested from the corresponding author.

\section{Consent to Publish:}

Not Applicable

\section{Ethics (and consent to participate):}

Not Applicable

\section{Funding:}

The project was funded through the research support for MRes students provided by Western Sydney University School of Science and Health and Graduate Research School.

\section{References}

1. Latge JP. Aspergillus fumigatus and aspergillosis. Clin Microbiol Rev. 1999;12(2):310-50. 
2. Baddley JW. Clinical risk factors for invasive aspergillosis. Med Mycol. 2011;49 Suppl 1:S7-S12.

3. Hagiwara D, Sakai K, Suzuki S, Umemura M, Nogawa T, Kato N, et al. Temperature during conidiation affects stress tolerance, pigmentation, and trypacidin accumulation in the conidia of the airborne pathogen Aspergillus fumigatus. PLoS One. 2017;12(5):e0177050.

4. Margalit $\mathrm{A}$, Kavanagh $\mathrm{K}$. The innate immune response to Aspergillus fumigatus at the alveolar surface. FEMS Microbiol Rev. 2015;39(5):670-87.

5. Wezensky SJ, Cramer RA, Jr. Implications of hypoxic microenvironments during invasive aspergillosis. Med Mycol. 2011;49 Suppl 1:S120-4.

6. Schrettl M, Beckmann N, Varga J, Heinekamp T, Jacobsen ID, Jochl C, et al. HapX-mediated adaption to iron starvation is crucial for virulence of Aspergillus fumigatus. PLoS Pathog. 2010;6(9):e1001124.

7. de Jager TL, Cockrell AE, Du Plessis SS. Ultraviolet Light Induced Generation of Reactive Oxygen Species. Adv Exp Med Biol. 2017;996:15-23.

8. Heinekamp T, Thywissen A, Macheleidt J, Keller S, Valiante V, Brakhage AA. Aspergillus fumigatus melanins: interference with the host endocytosis pathway and impact on virulence. Front Microbiol. 2012;3:440.

9. Ben-Ami R, Lamaris GA, Lewis RE, Kontoyiannis DP. Interstrain variability in the virulence of Aspergillus fumigatus and Aspergillus terreus in a Toll-deficient Drosophila fly model of invasive aspergillosis. Med Mycol. 2010;48(2):310-7.

10. Valero-Jimenez CA, Debets AJ, van Kan JA, Schoustra SE, Takken W, Zwaan BJ, et al. Natural variation in virulence of the entomopathogenic fungus Beauveria bassiana against malaria mosquitoes. Malar J. 2014;13:479.

11. Aufauvre-Brown A, Brown JS, Holden DW. Comparison of virulence between clinical and environmental isolates of Aspergillus fumigatus. Eur J Clin Microbiol Infect Dis. 1998;17(11):778-80.

12. Mondon P, De Champs C, Donadille A, Ambroise-Thomas P, Grillot R. Variation in virulence of Aspergillus fumigatus strains in a murine model of invasive pulmonary aspergillosis. J Med Microbiol. 1996;45(3):186-91.

13. Morton CO, Varga JJ, Hornbach A, Mezger M, Sennefelder H, Kneitz S, et al. The temporal dynamics of differential gene expression in Aspergillus fumigatus interacting with human immature dendritic cells in vitro. PLoS One. 2011;6(1):e16016.

14. Jamieson DJ. Saccharomyces cerevisiae has distinct adaptive responses to both hydrogen peroxide and menadione. J Bacteriol. 1992;174(20):6678-81. 
15. Paisley D, Robson GD, Denning DW. Correlation between in vitro growth rate and in vivo virulence in Aspergillus fumigatus. Med Mycol. 2005;43(5):397-401.

16. Amarsaikhan N, O'Dea EM, Tsoggerel A, Owegi H, Gillenwater J, Templeton SP. Isolate-dependent growth, virulence, and cell wall composition in the human pathogen Aspergillus fumigatus. PLoS One. 2014;9(6):e100430.

17. Rizzetto L, Giovannini G, Bromley M, Bowyer P, Romani L, Cavalieri D. Strain dependent variation of immune responses to A. fumigatus: definition of pathogenic species. PLoS One. 2013;8(2):e56651.

\section{Tables}

Table 1 Effect of acute exposure ( $3 \mathrm{~h}$ ) to menadione and $\mathrm{H}_{2} \mathrm{O}_{2}$ on the survival of conidia of clinical strains of $A$. fumigatus.

\begin{tabular}{|c|c|c|c|c|c|c|c|c|c|c|}
\hline \multirow[t]{3}{*}{ Isolate } & \multicolumn{5}{|c|}{ Menadione } & \multicolumn{5}{|c|}{$\mathrm{H}_{2} \mathrm{O}_{2}$} \\
\hline & \multicolumn{2}{|c|}{$30 \mathrm{mM}$} & \multicolumn{2}{|c|}{$50 \mathrm{mM}$} & \multirow[b]{2}{*}{ p-value ${ }^{b}$} & \multicolumn{2}{|c|}{$30 \mathrm{mM}$} & \multicolumn{2}{|c|}{$50 \mathrm{mM}$} & \multirow[b]{2}{*}{ p-value } \\
\hline & Mean $^{a}$ & SD & Mean & SD & & Mean & SD & Mean & SD & \\
\hline Af01 & 70.5 & 25.5 & 65.6 & 13.0 & 0.71 & 67.5 & 17.0 & 44.8 & 8.6 & 0.03 \\
\hline Af02 & 83.0 & 8.8 & 71.0 & 9.3 & 0.07 & 63.5 & 16.7 & 29.5 & 6.8 & 0.003 \\
\hline Af03 & 87.4 & 5.7 & 77.2 & 6.0 & 0.025 & 65.5 & 14.2 & 25.2 & 6.5 & 0.0004 \\
\hline Af04 & 79.7 & 13.4 & 72.2 & 12.7 & 0.39 & 63.6 & 11.8 & 40.1 & 19.8 & 0.06 \\
\hline Af05 & 76.8 & 13.2 & 60.8 & 12.6 & 0.086 & 73.8 & 5.6 & 18.9 & 4.2 & 0.0001 \\
\hline Af06 & 70.8 & 14.9 & 64.8 & 9.1 & 0.46 & 65.8 & 14.0 & 11.2 & 4.0 & 0.0001 \\
\hline Af07 & 76.4 & 12.7 & 68.1 & 12.8 & 0.34 & 43.9 & 7.6 & 6.5 & 2.4 & 0.0001 \\
\hline Af08 & 81.2 & 15.0 & 77.4 & 16.0 & 0.703 & 62.9 & 12.1 & 6.3 & 2.7 & 0.0001 \\
\hline Af09 & 87.6 & 3.9 & 79.6 & 3.5 & 0.0092 & 38.5 & 13.4 & 3.8 & 1.6 & 0.0004 \\
\hline Af10 & 88.3 & 8.2 & 78.3 & 5.8 & 0.06 & 37.3 & 8.1 & 3.5 & 1.0 & 0.0001 \\
\hline Af11 & 69.2 & 17.8 & 43.0 & 16.3 & 0.0414 & 46.7 & 16.1 & 0.6 & 0.6 & 0.0002 \\
\hline Af12 & 81.5 & 11.8 & 47.8 & 10.4 & 0.0014 & 70.3 & 18.9 & 4.1 & 3.6 & 0.0001 \\
\hline Af13 & 77.2 & 7.2 & 48.4 & 15.9 & 0.0062 & 48.0 & 16.8 & 0.7 & 0.8 & 0.0002 \\
\hline Af14 & 73.2 & 33.2 & 59.2 & 17.4 & 0.43 & 71.2 & 9.5 & 5.5 & 3.3 & 0.0001 \\
\hline Af15 & 77.1 & 7.4 & 53.9 & 8.5 & 0.0017 & 59.9 & 15.4 & 29.2 & 4.9 & 0.003 \\
\hline
\end{tabular}


a Mean of treatment relative to untreated control from five replicate experiments. ${ }^{\mathrm{b}} \mathrm{p}$-Value from t-test of $30 \mathrm{mM}$ data compared to $50 \mathrm{mM}$ data. Values in bold indicate isolates that showed no significant changes in inhibition between treatments with $30 \mathrm{mM}$ and $50 \mathrm{mM}$ of the oxidative stressor.

\section{Figures}

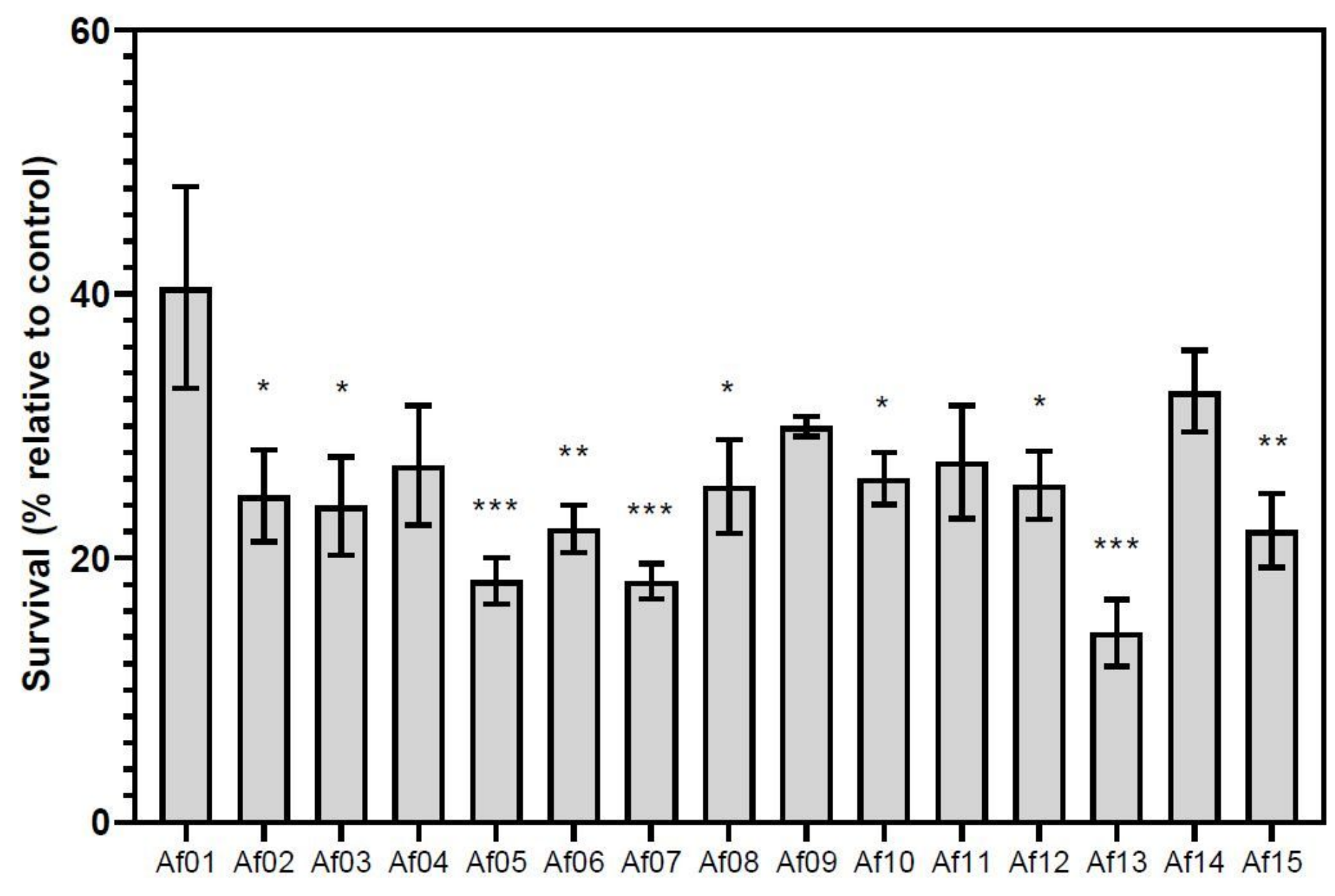

Aspergillus fumigatus isolates

Figure 1

Induction of oxidative damage in 15 isolates of A. fumigatus through exposure to UV light $(1.6 \mathrm{~W} / \mathrm{m} 2)$ for one minute. Data is expressed as percentage of CFUs surviving compared to untreated controls. Data represents mean and standard errors from five replicate experiments for each isolate. Data was analysed by one-way ANOVA with multiple comparison of all isolate means. 